\title{
Anti-Hypertensive Drugs and Sexual Dysfunction in Men
}

\author{
Noverio Tarukallo \\ Universitas Hasanuddin \\ Haerani Rasyid \\ Universitas Hasanuddin, haeraniabdurasyid@yahoo.com
}

\begin{abstract}
One of the risk factors associated with the incidence of sexual dysfunction in men is antihypertensive drugs. Anti-hypertensive drugs which have the effect of causing sexual dysfunction in men include; diuretics, clonidine, and $\beta$-blockers (except nebivolol), but there are some anti-hypertensive drugs that have a neutral effect, even have a positive effect which in this case can improve sexual function in men. Anti-hypertensive drugs that have a neutral effect on male sexual function include; Calcium Channel Blockers (CCBs)and Angiotensin Converting Enzyme Inhibitors (ACE-Inhibitors) and those that have the effect of increasing sexual function in men include; Angiotensin Receptor Blockers (ARBs) and $\beta$-blockers namely nebivolol. The use of anti-hypertensive drugs can affect sexual function in men through different mechanisms. Anti-hypertensive drugs such as diuretics, $\beta$-blockers, and clonidine can cause sexual dysfunction in men through the mechanism of changes in the sympathetic outflow, the effect on contractions of corporal smooth muscle, and through the influence on androgen hormone levels. ARBs and Nebivolol can improve sexual function through inhibition mechanism on Angiotensin II and increase the bioavailability of Nitric Oxide (NO).
\end{abstract}

Keywords: anti-hypertensive drugs, sexual dysfunction, men

\section{INTRODUCTION}

According to World Health Organization (WHO, 1975) sexual dysfunction is a condition where an individual cannot participate in a sexual intercourse as desired. Sexual dysfunction that occurs in men can be a decrease in libido, erectile dysfunction (ED), and problems with ejaculation. Epidemiological data indicate that erectile dysfunction has a high prevalence and incidence throughout the world. ${ }^{[1]}$ It is estimated that more than 152 million men worldwide experienced erectile dysfunction in 1995, and this number will increase by 170 million, which is estimated to reach 322 million by 2025. ${ }^{[2]}$ The study conducted by The National Social Life, Health and Aging Project (NSHAP) by taking subjects for men aged 57-85 years, the results of the incidence rate of erectile dysfunction were found to be $37 \%$ with an incidence rate of 26 new cases per 1000 men. Some risk factors associated with ED include; history of heart disease, hypertension, diabetes mellitus, smoking history, psychological conditions, hormone levels, especially testosterone, and drugs. ${ }^{[3]}$ Many drugs are known to affect sexual function in men, such as; anti-cholinergic, psychotropic, and anti-hypertensive drugs. ${ }^{[2]}$ 
Anti-hypertension therapy has been associated to the incidence of $\mathrm{ED},{ }^{[4,5]}$ from a study conducted by Doumas et al. (2013) found that the prevalence of ED is more common in hypertensive patients who are treated compared to hypertensive patients who are not treated and normotensive patients, this indicates that therapy for hypertension itself contributes to the occurrence of sexual dysfunction. ${ }^{[4,6]}$ The incidence rate of ED in patients taking anti-hypertensive drugs is $14 \% .^{[2]}$ One reason for the non-compliance with the use of anti-hypertensive drugs is because of the side effects which raisedthe occurrence of sexual dysfunction. The use of antihypertensive drugs can affect each sexual function with different mechanisms. ${ }^{[4]} \mathrm{It}$ has been proven that some antihypertensive drugs have the effect of causing sexual dysfunction such as diuretics, clonidine, and $\beta$-blockers especially those that are non-selective (except nebivolol), ${ }^{[1]}$ while other antihypertensive drugs (Calcium Channel Blockers, Angiotensin Converting Enzyme Inhibitors (ACE-Inhibitors)) have a neutral effect and even have a positive effect on sexual function in men with hypertension, whereas from several studies conducted, it was found that Angiotensin Receptor Blockers (ARBs) and nebivolol were associated with the increased of sexual function in men with hypertension. In this review, we will discuss about the relationship between anti-hypertensive drugs and the incidence of sexual dysfunction in male patients with hypertension.

\section{PHYSIOLOGY OF SEXUAL FUNCTION IN MEN}

The mechanism of erection in male sexual function includes 2 processes namely; the occurrence of dilatation of the arteriolar and the occurrence of increased blood flow to the tissues and blood vessels of the penis, where the innervation system that plays a role is the autonomic innervation system and the somatic innervation system. ${ }^{[2,24]}$ The mechanism of erection is also influenced by androgen hormones which are steroid hormones that play a role in controlling and maintaining erectile function in men. ${ }^{[25]}$

\section{Structure and innervation of male genitals}

The penis consists of two functional compartments, the corpus cavernosum and corpus spongiosum. Histologically, tissue from the corpus cavernosum consists of smooth muscle fibers that are interconnected with extracellular matrix tissue. The penis is innervated by the somatic and autonomic nervous system. The somatic neural system innervates the skeletal muscle from perineal, contraction of the skeletal muscle from perineal during 
erection causes a temporary increase in pressure in the corporeal area wherein it will help to increase tension of the penis. The autonomic innervation system innervates the penis through 2 innervation systems namely sympathetic innervation and parasympathetic innervation. Parasympathetic innervation plays a major role in erectile function and sympathetic innervation acts as mediation in the process of detumescence after orgasm, and if there is no sexual stimulation, sympathetic innervation helps maintaining the penis in a flaccid state. ${ }^{[2]}$

\section{Erectile and Flaccid Occurrence Mechanisms}

The erection begins with stimulation of the arousal center in the brain which will cause inhibition of the release of norepinephrine, parasympathetic stimulation which will induce the production and release of nitric oxide (NO), and stimulation of somatic nerves which will induce the production and release of acetylcholine. ${ }^{[11,21]}$ Nitric Oxide is produced by endothelium via Endothelial Nitric Oxide Synthase (eNOS) or neuronal via Neuronal Nitric Oxide Synthase (nNOS). Once released, NO will diffuse into the blood vessels and smooth muscles of the corpus cavernosum and will catalyze the change from Guanosine-5'Triphosphate (GTP) to Cyclic Guanosine Monophosphate (cGMP), which will result in activation of Protein Kinase $\mathrm{G}$ (PKG) which will cause relaxation of smooth muscle of the corpus cavernosum, then increased arterial inflow, relaxation of sinusoids, partial occlusion of venous outflow and finally erection. ${ }^{[13,15,21,28]}$ The cGMP level will be modulated by the Phosphodiesterase (PDE) enzyme, which will break down cGMP into an inactive form of 5GMP. ${ }^{[15]}$

The occurrence of a flaccid state of the penis is caused by an increase in levels of angiotensin (AT) II. Angiotensin II will cause biological effects through activation of Angiotensin AT1 receptors which modulate vasoconstriction by increasing calcium influx from extracellular to intracellular, Angiotensin II will also cause inflammation, activation of sympathetic innervation, and increased Reactive Oxygen Species (ROS) through activation of nicotinamide adenine dinucleotide phosphate (NADPH) oxidase. ${ }^{[15,28]}$ Norepinephrine is responsible for regulating smooth muscle tone of the corpus cavernosum through interactions with $\alpha-1$ receptors and $\alpha-2$ receptors. ${ }^{[2]}$ Reactive Oxygen Specieswill activate Rho-kinase pathway, which will prevent the dephosphorylation of miofilament which will cause contraction of smooth muscle, causing a flaccid state of the penis. ${ }^{[11,15,28]}$ 


\section{The Role of Androgen Hormones in} Male Sexual Function

Davidson et al. (1983) reported that there is a clear relationship between low androgen hormone levels and decreased sexual activity. Androgen hormones play a role in increasing the expression and activity of eNOS in the corpus cavernosum. ${ }^{[2,21]}$ Androgen hormones also play an important role in the development and maturation of Endothelial Progenitor Cells (EPCs), by stimulating the proliferation of EPCs through the Androgen Receptor/Vascular Endothelial Growth Factor (AR/VEGF)-mediated mechanism. EPCs have an important role in the process of repairing blood vessels, angiogenesis and replacement of damaged endothelial cells in blood vessels. ${ }^{[21]}$ In the state of endothelial dysfunction there is a decrease in expression of eNOS, increased production of ROS, increased synthesis and release of endothelin, increased production of Tumor Necrosis Factor $(\mathrm{TNF}-\alpha)$ and increased tone of blood vessels. ${ }^{[21]}$

\section{PATHOGENESIS OF SEXUAL DYSFUNCTION IN MEN}

The pathogenesis of the occurrence of sexual dysfunction is classified into several mechanisms, namely: Psychogenic, neurogenic, hormonal, vasculogenic, cellular, and iatrogenic mechanisms. ${ }^{[20]}$

\section{Psychogenic Mechanism}

Psychogenic conditions can cause sexual dysfunction in men. Depressive conditions can cause a decrease in libido and disruption in releasing NO. Several factors that play a role in the occurrence of psychogenic sexual dysfunction, are shown in Table 1. ${ }^{[20]}$

\section{Neurogenic Mechanism}

Diseases or conditions that cause disorders of the central nervous system and peripheral nervous system can cause sexual function disorders, in the form of erectile dysfunction, and difficulty reaching orgasm. ${ }^{[20,22]}$ Neurons in the cortex, thalamus, hypothalamus, midbrain and pons, play an important role in the function of ejaculation. ${ }^{[2]}$ Multiple sclerosis is a disease that affects the central nervous system, which can cause ED, a condition that occurs due to interference with the hypothalamus-pituitary-testis axis which will reduce the levels of androgen hormones. ${ }^{[22]}$

Erectile function is controlled by parasympathetic nerve fibers from S2-S4. These nerve fibers will travel through the pelvicus nerve and pelvicus plexus to the cavernous nerve, which enters the corpus cavernosum. when activation occurs, the cavernous nerve will release $\mathrm{NO}$ and acetylcholine. Trauma in the pelvis can cause ED and ejaculation disorders due to damage to the pelvicus nerve. The 
ejaculatory reflex is coordinated by the spinal cord and depends on thoracolumbar nerve fibers from the T10-L2 segment and somatic nerve fibers as high as S2S4.Injuries to the spinal cord can cause sexual dysfunction in the form of ED and ejaculation dysfunction. ${ }^{[22]}$

\section{Hormonal Mechanism}

Testosterone hormone has a very important role in sexual function in men, a decrease in the level of androgen testosterone hormone will cause a decrease and loss of libido and ED. ${ }^{[3,20]}$

Circumstances that can cause a decrease in testosterone levels, divided into two parts namely primary hypogonadism, where there is problems in the testis and hypogonadotropic hypogonadism, where primary disease affects the pituitary and hypothalamus, ${ }^{[20]}$ like in hyperprolactinemia condition, where there is a decrease expression of Gonadotropin-Releasing Hormone $(\mathrm{GnRH})$, a decrease GnRH activity in the pituitary, a decrease secretion of $\mathrm{GnRH}$, and conversion hindrance from testosterone to an active metabolite, dihydrotestosterone. ${ }^{[2]}$

\section{Vasculogenic Mechanism}

Impaired blood vessels can cause sexual dysfunction, through a reduction in arterial inflow that will cause a relative hypoxic state in the penis, which will cause an increase of transforming growth factor beta, that will induce changes in the smooth cavernosum muscle of the penis. ${ }^{[20]}$ Some conditions that can cause a decrease in arterial supply and venous drainage of the penis are shown in Table3. $[2,20]$

\section{Cellular Mechanism}

There are 2 types of cavernous cells that have an important role in erectile function, namely smooth muscle cells and endothelial cells. It is known that there are several conditions that can cause endothelial dysfunction, including; atherosclerosis, hypertension, hypercholesterolemia, diabetes mellitus and smoking. ${ }^{[3]}$ In conditions of endothelial dysfunction there is a decrease in the endothelium-dependent smooth muscle relaxation process caused by loss and increase in destruction of NO. ${ }^{[1,3,20]}$

\section{Iatrogenic Mechanism}

A large number of drugs can interfere with sexual function, which include disturbing erectile function, ejaculatory function and libido. ${ }^{[20]}$ Some drugs such as; anti-androgen groups, ketoconazole and progestins, are known to change the hypothalamic-pituitarytesticular axis and cause disruption to the production and work of the testosterone hormone. Benzodiazepine drugs have a sedative effect, which will cause a decrease in libido. Antidepressants can cause sexual dysfunction through the 
mechanism of 5-HT2 receptor activation, which will inhibit noradrenergic and dopaminergic transmission and also cause sexual dysfunction through hypothalamicpituitary-adrenal (HPA) dysregulation Axis. Dopaminergic neurotransmission plays a role in male sexual function through the role of the erection process, where dopamine plays a role in regulating the synthetic increase of NO. ${ }^{[2]}$

\section{RELATIONSHIP OF ANTI- HYPERTENSIVE DRUGS AND SEXUAL DISFUNCTION IN MEN}

Anti-hypertensive drugs such as diuretics, $\beta$-blockers except nebivolol, and clonidine are known to cause erectile dysfunction by changing the sympathetic outflow, affecting contractions of corporal smooth muscle, and affecting the levels of androgen hormones. ${ }^{[14]}$ Some other antihypertensive drugs such as ARBs, ACEinhibitors, and Calcium Channel Blockers can improve sexual function through an inhibitory mechanism in Angiotensin II and increase the bioavailability of NO. ${ }^{[10,12,14]}$

\section{Angiotensin Receptor Blockers}

Some studies state that ARBs can improve sexual function in men. ${ }^{[4,7,9,15]}$ Based on study conducted by Manolis et al (2012), it was said that replacing antihypertensive drugs with a class of ARBs can restore the occurred state of erectile dysfunction. Ferrario et al. (2002) reported that ARBs, namely Losartan and Valsartan, reported reducing the incidence of erectile dysfunction in men with hypertension and consistently improving erectile function. ARBs had a positive effect on erectile function through inhibiting Angiotensin II. ${ }^{[14]}$ Studies show that anti-hypertension ARBs improve endothelial function in the cavernosum network, ${ }^{[10,14,15]}$ this is based on the role of angiotensin II which is known to induce contraction of the penile corpus cavernosum smooth muscle and induce endothelial dysfunction through activation and increase in reactive oxygen species through bonding AT I receptor, so there is a decrease in NO synthesis, ROS will also react with $\mathrm{NO}$ and form $\mathrm{ONOO}^{-}$, so there is a decrease in the effectiveness of NO. ${ }^{[7,14]}$

\section{ACE-Inhibitors}

Some studies say that ACEinhibitors do not cause sexual dysfunction and can even improve sexual function in men. ${ }^{[10,12]}$ The association between ACEinhibitors drug use in the absence of complications of sexual dysfunction has been demonstrated in some study. ${ }^{[5]}$ One explanation that can explain this situation is that ACE inhibitors will inhibit ACE, resulting in decreased levels of angiotensin II so that the role of angiotensin II in the renin-aldosterone system that induced 
vascular remodeling process will be inhibited and will also increase the erectile response. ${ }^{[5,16]}$ Croog et al. (1986) found that the use of captopril had an effect on improving sexual function in men, this was also supported by studies conducted by $\mathrm{Di}$ Bianco et al. (1991), which stated that 40$80 \%$ of male patients treated with ACEinhibitors experienced increased sexual function. ACE-inhibitors can also improve erectile function in men with hypertension by increasing levels of bradykinin which has the effect of inducing relaxation of the smooth muscle of the corpus cavernosum.

\section{Clonidine}

Anti-hypertension drugs $\quad \alpha 2-$ adrenergic agonist namely Clonidine, are reported to cause ED. ${ }^{[2,8,13,14]}$ Dong Wang et al (2015) reported that the use of clonidine in addition to causing ED can even reduce the level of conception. ${ }^{[16]}$ The use of Clonidine can cause ED through mechanism that reduce the sympathetic outflow and induce the contraction of the corporal smooth muscle. ${ }^{[14]}$

\section{4. $\beta$-Blockers}

Erectile dysfunction is a common side effect as a result of the use of $\beta$ blockers. ${ }^{[3,5,7,7,15]}$ In an international survey conducted, it was found that $20 \%$ of men taking $\beta$-blockers experience erectile dysfunction. ${ }^{[10]}$ The use of $\beta$-blockers, namely atenolol, metoprolol, carvedilol, and propranolol has been associated with an increased incidence of sexual dysfunction. ${ }^{[4]}$ Administration of $\beta$ blockers causes a significant decrease in testosterone levels plasma and Luteinizing hormone which causes a decrease in libido, this indicates that $\beta$-blockers can cause sexual dysfunction through direct influence on sex hormone production. ${ }^{[1,14,15]} \beta$-blockers have also been reported to cause erectile dysfunction by potentiating the alpha-1 adrenoreceptor-mediated mechanism in inducing contractions of the corporal penile smooth muscle. ${ }^{[5,7,14]}$ In contrast to other groups of $\beta$-blockers, groups of $\beta$-blockers selective $\beta-1$ namely Nebivolol can improve sexual function and improve the state of previous sexual dysfunction in male patients with hypertension by increasing endothelial function, increasing bioavailability of $\mathrm{NO}$, having antioxidant effects, inhibiting proliferation of smooth muscle cells in blood vessels and increasing expression of eNOS. ${ }^{[4,6,10,15,26,27,29]}$

\section{Calcium Channel Blockers}

The calcium channel blockers (CCBs) from several studies have been shown not causing sexual dysfunction and even have a positive effect on sexual function. ${ }^{[6,10,12]}$ Several studies report that CCBs anti-hypertensive drugs can improve endothelial function. The calcium channel blockers work on T-type calcium channels 
on microvascular endothelial cells and induce NO formation through activation of the enzyme nitric oxide synthase. Groups of CCBs also in several studies have reported to have anti-atherosclerotic effects, Matsubara et al (2008) reported that the use of CCBs can cause suppression of vascular cell adhesion molecule (VCAM)-1, intracellular cell adhesion molecule-1 (ICAM-1). Roth et al reported that the use of groups of CCBs namely, amlodipine, verapamil and diltiazem significantly reduced plateletderived growth factor $\beta$-dependent collagen deposition in the extracellular matrix produced by smooth muscle cells from blood vessels and fibroblast cells.

\section{Diuretics}

Diuretic groups have side effects that can cause sexual dysfunction. ${ }^{[9,10,12]}$ Erectile dysfunction is a common side effect, either as a single or combination therapy where diuretics are additional therapy. ${ }^{[13]}$ From research conducted by Rastogi et al. (2007), it is reported that 40$60 \%$ of patients taking thiazide diuretic experience a decrease in sexual function. The use of thiazide can cause disruption of secretions from Luteinizing Hormone and Testosterone, and cause Zinc deficiency. Use of diuretic therapy, spironolactone can cause ED and decrease in libido caused by secondary effects of spironolactone as anti-androgens that inhibit peripheral receptors androgen and inhibits dihydrotestosterone binding. ${ }^{[2,5]}$

\section{CONCLUSION}

There is a relationship between antihypertensive therapy and incidence of sexual dysfunction in men with hypertension. Erection occurs due to activation of parasympathetic innervation and stimulation of somatic nerves which will stimulate increased production and activity of NO that will cause relaxation of smooth muscles of the corpus cavernosum and cavernosum blood vessels, increased arterial inflow, sinusoid relaxation, and passive blood restriction through activation of PKG. The flaccid state of the penis is caused by an increase in angiotensin II levels and an increase in ROS, where both conditions will cause contractions of smooth muscle.

The pathogenesis of sexual dysfunction is classified in several mechanisms, namely: psychogenic, neurogenic, hormonal, vasculogenic, cellular, and iatrogenic mechanisms. Diuretics, clonidine and $\beta$-Blockers except nebivolol can cause sexual dysfunction in patients with hypertension, each with a different mechanism. ARBs, ACE inhibitors, Calcium Channel Blockers, and nebivolol do not cause sexual dysfunction and even have a positive effect on sexual 
function in men with hypertension, each with different mechanisms.

\section{REFERENCES}

1. Hatzimouratidis $\mathrm{K}$, Eardley I, Giuliano F, et al. Guidelines on Male Sexual Dysfunction: Erectile Dysfunction and Premature Ejaculation. EAU.2015:p2-10.DOI: 10.1016/j.eururo.2010.02.020.

2. Kandeel FR, Koussa KT, Swerdloff.Male Sexual Function and Its Disorders: Physiology, Pathophysiology, Clinical Investigation, and Treatment. Endocr Rev. 2001; 22(3):342-88. DOI: 10.1210/edrv.22.3.0430

3. Chiesa AD, Ptiffner D, Meier B, et al. Sexual Activity in Hypertensive Men. JHum Hypertens.2003;17: 51521.DOI:10.1038/sj.jhh. 1001580

4. Manolis A, Doumas M.Antihypertensive Treatment and Sexual Dysfunction. CurrHypertens Rep.2012;14:285-92.

DOI: 10.1007/s11906-012-0276-5

5. Spatz ES.Sexual Activity and Function among Middle-Aged and Older Men and Women with Hypertension.

Hypertens.2013;31(6):1-11. DOI: 10.1097/HJH.0b013e32835fdefa

6. Javaroni V, Neves MF.Erectile Dysfunction and Hypertension:Impact on Cardiovascular Risk and Treatment.Int J Hypertens. 2012:p; 111.DOI: $10.1155 / 2012 / 627278$

7. Yang L, Yu J, Ma R, Lin X, et al.The Effect of Combined Antihypertensive Treatment (Felodipine with Either Irbesartan or Metoprolol) on Erectile Function: A Randomized Controlled Trial.Cardiol J.2013;125:235-41. DOI: $10.1159 / 000350955$

8. Schiffrin EL.Circulatory Therapeutics:Use of Antihypertensive Agents and Their Effects on The Vasculature.JCell
Med.2010;5(14):1018-29. DOI: 10.1111/j.1582-4934.2010.01056.x

9. Ekman E, Haag S, Sundstrom S, et al. Antihypertensive Drugs and Erectile Dysfunction as seen in Spontaneous Reports, With Focus on Angiotensin II Type I Receptor Blockers.DrugHealthcPatient Saf. 2010; 2: 21-5.

10. Conaglen HM, Conaglen JV. DrugInduced Sexual Dysfunction in Men and Women. Aust Prescr.2013;36:425. DOI: 10.18773/austprescr.2013.021

11. Dean RC, lue TF. Physiology of Penile Erection and Pathophysiology of Erectile Dysfunction. Urol Clin North Am.2005;32(4):379-385.DOI: 10.1016/j.ucl.2005.08.007

12. Vlachopoulos C, Jackson G, Stefanadis Cet al.Erectile Dysfunction in the Cardiovascular Patient. EurHeart J. 2013;34:2034-46. DOI: 10.1093/eurheartj/eht112

13. Ryu JK, Cho KS, Kam SC et al. Korean Society for Sexual Medicine and Andrology (KSSMA) Guideline on Erectile Dysfunction.World J Mens Health.2013;31(2):83-102. DOI: 10.5534/wjmh.2013.31.2.83

14. La Torre A, Glupponi G, Duffy D et al.Sexual Dysfunction Related To Drugs: A Critical Review. Pharmacopsychiatry.2015;48:1-6. DOI: $10.1055 / \mathrm{s}-0034-1395515$

15. Nunes KP, Labazi H, Webb RC. New Insight into Hypertension-Associated Erectile

Dysfunction.CurrOpinNephrol

Hypertens.2012; 21(2):163-170. DOI: 10.1097/MNH.0b013e32835021bd

16. Wang D, Wang W, Liu AJ et al.LongTerm Treatment of Clonidine, Atenolol, Amlodipine and Hydrochlorothiazide, but Not Enalapril, Impairs the Sexual Function in Male Spontaneously Hypertensive Rats. Plos One.2015;3:1-13. DOI: 10.1371/journal.pone.0116155

17. Nunes KP, Webb RC. Mechanism in Erectile Function and Dysfunction: An 
Overview, Erectile Dysfunction Disease Associated Mechanisms and Novel Insights into Therapy. In Techopen.2012;3:1-21.

18. Ferrario CM, Levy P. Sexual Dysfunction in Patients with Hypertension: Implication for Therapy.J

ClinHypertens.2002;6(4):424-432.

19. Salman M, Shehzadi N, Khan MT et al. Erectile Dysfunction: prevalence, risk factors and involvement of antihypertensive drugs intervention.TropJPharmRes.2016;15
(4):869-76.
DOI:
10.4314/tjpr.v15i4.29

20. Eardley I. Pathophysiologyof Erectile Dysfunction. $\mathrm{Br} \mathrm{J}$ Diabetes Vasc Dis.2002;4(2): 272-76. DOI: 10.1177/14746514020020040701

21. Traish A M, Galoosian A.Androgens Modulate Endothelial Function and Endothelial Progenitor Cells in Erectile Physiology.Korean J Urol.2013; 54:721-31. DOI: 10.4111/kju.2013.54.11.721

22. Antwerp J.Neurogenic Sexual Dysfunction. $2^{\text {nd }}$ Int Neuro-Urology Meeting. 2013; 2:1-4.

23. Fode M, Brackett NL, Ohl DA et al. Male Sexual Dysfunction And Fertility Associated With
Neurological Disorders. Asian JAndrol.2012; 14:61-8. DOI: 10.1038/aja.2011.70

24. Booth AM. Physiology of Male Sexual Function. Ann Intern Med.1980;92: 329-331.

25. Miller WL. The Molecular Biology, Biochemistry, and Physiology of Human Steroidogenesis and its Disorders. Endocr Rev.2011;32(1):81151. DOI: 10.1210/er.2010-0013

26. Weiss R. Nebivolol: A Novel BetaBlocker with Nitric Oxide-Induced Vasodilatation. Vasc Health Risk Manag. 2006;2(3):303-8.

27. Basile JN. The Role of Vasodilating $\beta$-blockers in controlling Hypertension as a means of Reducing Cardiovascular and Stroke Risk. American J Med.2010;7(123):S9-S15. DOI: 10.1016/j.amjmed.2010.04.013

28. Fraga Silva RA, Montecucco F, Mach $F$ et al. Pathophysiology Role of the Renin-Angiotensin System on Erectile Dysfunction. Eur J Clin Invest.2013;7:1-9. DOI: 10.1111/eci.12117

29. Maffei A, Lembo G.Nitric Oxide mechanisms of Nebivolol.Ther Adv Cardiovasc Dis.2009;3(4):317-327. DOI: $10.1177 / 1753944709104496$

\section{LIST OF TABLES}

Table 1. Contributing factors of psychogenic sexualdysfunction. ${ }^{20}$

\begin{tabular}{ll}
\hline \hline Predisposing & Educational issues (e.g. poor sexual education) \\
factors & Cultural issues (e.g. restricted upbringing \\
& Traumatic Sexual Experience \\
& Lifestyle issues (e.g. marital, financial stress) \\
Precipitating & Organic disorders \\
Factors & Extra-marital affairs \\
& Unreasonable expectations \\
& Depression and anxiety \\
Maintaining & Loss of Partner (e.g. "widower's syndrome”) \\
factors & Performance Related Anxiety \\
& Diminished attraction to partner \\
& Educational issues (e.g. poor sexual education) \\
\hline
\end{tabular}


Table 2. Diseases that can cause neurogenic sexualdysfunction. ${ }^{20}$

\begin{tabular}{ll}
\hline \hline Diseases of the & Multiple Sclerosis \\
central nervous & Spinal Cord Injury \\
system & Depression \\
& $\begin{array}{l}\text { Parkinson's disease } \\
\text { Cerebrovascular disease }\end{array}$ \\
Diseases of the & Cauda Equina compression \\
peripheral & Prolapsed inter-vertebral disc \\
nervous system & Peripheral neuropathy (e.g. diabetes, alcohol) \\
& Surgical Injury to Pelvic Nerves \\
\hline
\end{tabular}

Table 3.Vascular causes of sexual dysfunction in men. ${ }^{20}$

\begin{tabular}{ll}
\hline \hline Type of condition & Examples \\
Arterial Disorders & Pelvic Fracture associated arterial injury \\
& $\begin{array}{l}\text { Radiotherapy to pelvic malignancy } \\
\text { Aorto-iliac surgery }\end{array}$ \\
Abnormal Venous Channels & $\begin{array}{l}\text { Primary (congenital) } \\
\text { Following surgery for priapism }\end{array}$ \\
Failure toveno-occlusive & Following repeated urethral surgery \\
mechanism & $\begin{array}{l}\text { Abnormal tunical function (e.g. due to aging, } \\
\text { peyronie's } \\
\text { Reduced or abnormal smooth muscle (e.g. } \\
\text { due to aging, renal failure) }\end{array}$ \\
\hline
\end{tabular}

\section{LIST OF FIGURES}

1. Figure 1.Male Genital Anatomy. ${ }^{2}$

2. Figure 2.Penis Erection Mechanism. ${ }^{11}$

3. Figure 3. Flaccid Mechanismin Penis. ${ }^{17}$

4. Figure 4.The Role of Androgen Hormones in Regulating Endothelial Function. ${ }^{21}$

5. Figure5.Mechanism of sexual dysfunction caused by endothelial dysfunction. ${ }^{3}$

6. Figure6. ARBs Mechanism in Improving Sexual Function in Men ${ }^{18}$ 